\title{
THE ASYMPTOTIC BEHAVIOUR OF HEEGAARD GENUS
}

\author{
MARC LACKENBY
}

\section{Introduction}

Heegaard splittings have recently been shown to be related to a number of important conjectures in 3-manifold theory: the virtually Haken conjecture, the positive virtual $b_{1}$ conjecture and the virtually fibred conjecture [3]. Of particular importance is the rate at which the Heegaard genus of finite-sheeted covering spaces grows as a function of their degree. This was encoded in the following definitions.

Let $M$ be a compact orientable 3 -manifold. Let $\chi_{-}^{h}(M)$ be the negative of the maximal Euler characteristic of a Heegaard surface for $M$. Let $\chi_{-}^{s h}(M)$ be the negative of the maximal Euler characteristic of a strongly irreducible Heegaard surface for $M$, or infinity if such a surface does not exist. Define the infimal Heegaard gradient of $M$ to be

$$
\inf _{i}\left\{\frac{\chi_{-}^{h}\left(M_{i}\right)}{d_{i}}: M_{i} \text { is a degree } d_{i} \text { cover of } M\right\} .
$$

The infimal strong Heegaard gradient of $M$ is

$$
\liminf _{i}\left\{\frac{\chi_{-}^{s h}\left(M_{i}\right)}{d_{i}}: M_{i} \text { is a degree } d_{i} \text { cover of } M\right\} .
$$

The following conjectures were put forward in [3]. According to Theorem 1.7 of [3], either of these conjectures, together with a conjecture of Lubotzky and Sarnak [4] about the failure of Property $(\tau)$ for hyperbolic 3-manifolds, would imply the virtually Haken conjecture for hyperbolic 3-manifolds.

Heegaard gradient conjecture. A compact orientable hyperbolic 3-manifold has zero infimal Heegaard gradient if and only if it virtually fibres over the circle. Strong Heegaard gradient conjecture. Any closed orientable hyperbolic 3-manifold has positive infimal strong Heegaard gradient.

Some evidence for these conjectures was presented in [3]. More precisely, suitably phrased versions of these conjectures were shown to be true when one restricts attention to cyclic covers dual to a non-trivial element of $H_{2}(M, \partial M)$, to reducible manifolds and (in the case of the strong Heegaard gradient conjecture) to congruence covers of arithmetic hyperbolic 3-manifolds.

Received November 13, 2002. 
A less quantitative version of the conjectures is simply that $\chi_{-}^{\text {sh }}\left(M_{i}\right)$ cannot grow too slowly as a function of $d_{i}$, and that if $\chi_{-}^{h}\left(M_{i}\right)$ does grow sufficiently slowly, then $M$ is virtually fibred. These expectations are confirmed in the following result, which is the main theorem of this paper.

Theorem 1. Let $M$ be a closed orientable 3-manifold that admits a negatively curved Riemannian metric. Let $\left\{M_{i} \rightarrow M\right\}$ be a collection of finite regular covers with degree $d_{i}$.

(1) If $\chi_{-}^{h}\left(M_{i}\right) / \sqrt{d_{i}} \rightarrow 0$, then $b_{1}\left(M_{i}\right)>0$ for all sufficiently large $i$.

(2) $\chi_{-}^{s h}\left(M_{i}\right) / \sqrt{d_{i}}$ is bounded away from zero.

(3) If $\chi_{-}^{h}\left(M_{i}\right) / \sqrt[4]{d_{i}} \rightarrow 0$, then $M_{i}$ fibres over the circle for all sufficiently large $i$.

A slightly weaker form of Theorem 1(1) appeared in [3] as Corollary 1.4, with essentially the same proof. It is included here in order to emphasise its connection to the other two results.

The following corollary of Theorem 1(3) gives a necessary and sufficient condition for $M$ to be virtually fibred in terms of the Heegaard genus of its finite covers. We say that a collection $\left\{M_{i} \rightarrow M\right\}$ of finite covers has bounded irregularity if the normalisers of $\pi_{1} M_{i}$ in $\pi_{1} M$ have bounded index in $\pi_{1} M$.

Corollary 2. Let $M$ be a closed orientable 3-manifold with a negatively curved Riemannian metric, and let $\left\{M_{i} \rightarrow M\right\}$ be its finite-sheeted covers with degree $d_{i}$. Then the following are equivalent:

(1) $M_{i}$ is fibred for infinitely many $i$;

(2) in some subsequence with bounded irregularity, $\chi_{-}^{h}\left(M_{i}\right)$ is bounded;

(3) in some subsequence with bounded irregularity, $\chi_{-}^{h}\left(M_{i}\right) / \sqrt[4]{d_{i}} \rightarrow 0$.

Proof. (1) $\Rightarrow(2)$. If some finite-sheeted cover $\tilde{M}$ is fibred, then so is any finite cyclic cover $M_{i}$ of $\tilde{M}$ dual to the fibre. The normaliser $N\left(\pi_{1} M_{i}\right)$ of $\pi_{1} M_{i}$ in $\pi_{1} M$ contains $\pi_{1} \tilde{M}$, so $\left[\pi_{1} M: N\left(\pi_{1} M_{i}\right)\right]$ is bounded and hence these covers have bounded irregularity. Also, $\chi_{-}^{h}\left(M_{i}\right)$ is bounded by twice the modulus of the Euler characteristic of the fibre, plus four.

$(2) \Rightarrow(3)$. This is trivial, since $d_{i}$ must tend to infinity.

$(3) \Rightarrow(1)$. Since $\left[\pi_{1} M: N\left(\pi_{1} M_{i}\right)\right]$ is bounded in this subcollection, we may pass to a further subsequence where $N\left(\pi_{1} M_{i}\right)$ is a fixed subgroup of $\pi_{1} M$. Let $\tilde{M}$ be the finite-sheeted cover of $M$ corresponding to this subgroup. Then, the covers $\left\{M_{i} \rightarrow M\right\}$ in this subsequence give a collection $\left\{M_{i} \rightarrow \tilde{M}\right\}$ of finite regular covers such that $\chi_{-}^{h}\left(M_{i}\right) /\left[\pi_{1} \tilde{M}: \pi_{1} M_{i}\right]^{1 / 4} \rightarrow 0$. By Theorem $1(3), M_{i}$ is fibred for all sufficiently large $i$.

\section{Background material}

\section{Generalised Heegaard splittings}

A Heegaard splitting of a closed orientable 3-manifold can be viewed as arising from a handle structure. If one builds the manifold by starting with a single 0handle, then attaching some 1-handles, then some 2-handles and then a 3-handle, 
the manifold obtained after attaching the 0- and 1-handles is handlebody, as is the closure of its complement. Thus, the boundary of this submanifold is a Heegaard surface. Generalised Heegaard splittings arise from more general handle structures: one starts with some 0-handles, then adds some 1-handles, then some 2-handles, then 1-handles, and so on, in an alternating fashion, ending with some 3 -handles. One then considers the manifold embedded in $M$ consisting of the 0-handles and the first $j$ batches of 1 - and 2-handles. Let $F_{j}$ be the boundary of this manifold, but discarding any 2 -sphere components that bound 0- or 3-handles. After a small isotopy, so that these surfaces are all disjoint, they divide $M$ into compression bodies. In fact, the surfaces $\left\{F_{j}: j\right.$ odd $\}$ form Heegaard surfaces for the manifold $M-\bigcup\left\{F_{j}: j\right.$ even $\}$. We term the surfaces $F_{j}$ even or odd, depending on the parity of $j$.

More details about generalised Heegaard splittings can be found in [7] and [6]. The following theorem summarises some of the results from [7].

Theorem 3. From any minimal genus Heegaard surface $F$ for a closed orientable irreducible 3-manifold $M$, other than $S^{3}$, one can construct a generalised Heegaard splitting $\left\{F_{1}, \ldots, F_{n}\right\}$ in $M$ with the following properties:

1. $F_{j}$ is incompressible and has no 2-sphere components, for each even $j$;

2. $F_{j}$ is strongly irreducible for each odd $j$;

3. $F_{j}$ and $F_{j+1}$ are not parallel for any $j$;

4. $\left|\chi\left(F_{j}\right)\right| \leq|\chi(F)|$ for each $j$;

5. $|\chi(F)|=\sum(-1)^{j} \chi\left(F_{j}\right)$.

Corollary 4. Let $M, F$ and $\left\{F_{1}, \ldots, F_{n}\right\}$ be as in Theorem 3. Suppose that, in addition, $M$ is not a lens space. Let $\bar{F}$ be the surface obtained from $\bigcup_{j} F_{j}$ by replacing any components that are parallel by a single component. Then

1. $|\chi(\bar{F})| \leq\left|\chi\left(\bigcup_{j} F_{j}\right)\right|<|\chi(F)|^{2}$;

2. $\bar{F}$ has at most $\frac{3}{2}|\chi(F)|$ components.

Proof. Note first that no component of $\bigcup_{j} F_{j}$ is a 2 -sphere. When $j$ is even, this is (1) of Theorem 3. The same is true when $j$ is odd, since the odd surfaces form Heegaard surfaces for the complement of the even surfaces, and $M$ is not $S^{3}$. Hence, none of the compression bodies $H$ in the complement of $\bigcup_{j} F_{j}$ is a 3-ball.

We claim also that no $H$ is a solid torus. For if it were, consider the compression body to which it is adjacent. If this were a product, some even surface would be compressible, contradicting (1) of Theorem 3 . However, if it was not a product, then it is a solid torus, possibly with punctures. If it has no punctures, then $M$ is a lens space, contrary to assumption. If it does, then some component of an even surface would be a 2 -sphere, again contradicting (1). This proves the claim.

We expand (5) of Theorem 3 as follows:

$$
|\chi(F)|=\frac{-\chi\left(F_{1}\right)}{2}+\frac{\chi\left(F_{2}\right)-\chi\left(F_{1}\right)}{2}+\frac{\chi\left(F_{2}\right)-\chi\left(F_{3}\right)}{2}+\cdots+\frac{-\chi\left(F_{n}\right)}{2} .
$$


For any compression body $H$, other than a 3-ball, with negative boundary $\partial_{-} H$ and positive boundary $\partial_{+} H, \chi\left(\partial_{-} H\right)-\chi\left(\partial_{+} H\right)$ is even and non-negative. It is zero if and only if $H$ is a product or a solid torus. Since $F_{j}$ and $F_{j+1}$ are not parallel for any $j$, each term in $(*)$ is therefore at least one. So, $n+1$, the number of terms on the right-hand side of $(*)$, is at most $|\chi(F)|$. Hence,

$$
\left|\chi\left(\bigcup_{j} F_{j}\right)\right|=\sum_{j}\left|\chi\left(F_{j}\right)\right| \leq n|\chi(F)|<|\chi(F)|^{2} .
$$

The inequality $|\chi(\bar{F})| \leq\left|\chi\left(\bigcup_{j} F_{j}\right)\right|$ simply follows from the fact that we discard some components of $\bigcup_{j} F_{j}$ to form $\bar{F}$. This proves (1).

Now it is trivial to check that, for any compression body $H$, other than a 3 -ball, solid torus or product, $|\partial H| \leq \frac{3}{2}\left(\chi\left(\partial_{-} H\right)-\chi\left(\partial_{+} H\right)\right)$. The number of components of $\bar{F}$ is half the sum, over all complementary regions $H$ of $\bigcup_{j} F_{j}$ that are not products, of $|\partial H|$. This is at most $\frac{3}{4}\left(\chi\left(\partial_{-} H\right)-\chi\left(\partial_{+} H\right)\right)$. But the sum, over all complementary regions $H$ of $\bigcup_{j} F_{j}$, of $\frac{1}{2}\left(\chi\left(\partial_{-} H\right)-\chi\left(\partial_{+} H\right)\right)$ is the right-hand side of $(*)$. Thus, we deduce that the number of components of $\bar{F}$ is at most $\frac{3}{2}|\chi(F)|$, proving $(2)$.

\section{Realisation as minimal surfaces}

One advantage of using generalised Heegaard splittings satisfying (1) and (2) of Theorem 3 is that minimal surfaces then play a rôle in the theory. The following theorem of Freedman, Hass and Scott [2] applies to the even surfaces. Theorem 5. Let $S$ be an orientable embedded incompressible surface in a closed orientable irreducible Riemannian 3-manifold. Suppose that no two components of $S$ are parallel, and that no component is a 2-sphere. Then there is an ambient isotopy of $S$ so that afterwards each component is either a least area, minimal surface or the boundary of a regular neighbourhood of an embedded, least area, minimal non-orientable surface.

We will apply the above result to the incompressible components of $\bar{F}$. If we cut $M$ along these components, the remaining components form strongly irreducible Heegaard surfaces for the complementary regions. A theorem of Pitts and Rubinstein [5] now applies.

Theorem 6. Let $S_{1}$ be a (possibly empty) embedded stable minimal surface in a closed orientable irreducible Riemannian 3-manifold $M$ with a bumpy metric. Let $S_{2}$ be a strongly irreducible Heegaard surface for a complementary region of $S_{1}$. Then there is an ambient isotopy, leaving $S_{1}$ fixed, taking $S_{2}$ to a minimal surface, or to the boundary of a regular neighbourhood of a minimal embedded non-orientable surface, with a tube attached that is vertical in the I-bundle structure on this neighbourhood.

Bumpy metrics were defined by White in [8]. After a small perturbation, any Riemannian metric can be made bumpy. Then we may ambient isotope $\bar{F}$ so that each component is as described in Theorems 5 and 6 . 
We will need some parts of the proof of Theorem 6 , and not just its statement. Let $X$ be the component of $M-S_{1}$ containing $S_{2}$. Then, as a Heegaard surface, $S_{2}$ determines a sweepout of $X$. In any sweepout, there is a surface of maximum area, although it need not be unique. Let $a$ be the infimum, over all sweepouts in this equivalence class, of this maximum area. Then Pitts and Rubinstein showed that there is a sequence of sweepouts, whose maximal area surfaces tend to an embedded minimal surface, and that the area of these surfaces tends to $a$. This minimal surface, or its orientable double cover if it is non-orientable, is isotopic to $S_{2}$ or to a surface obtained by compressing $S_{2}$.

Now, when $M$ is negatively curved, one may use Gauss-Bonnet to bound the area of this surface. Suppose that $\kappa<0$ is the supremum of the sectional curvatures of $M$. Then, as the surface is minimal, its sectional curvature is at most $\kappa$. Hence, by Gauss-Bonnet, its area is at most $2 \pi\left|\chi\left(S_{2}\right)\right| /|\kappa|$. Thus, we have the following result.

Addendum 7. Let $S_{1}, S_{2}$ and $M$ be as in Theorem 6. Suppose that the sectional curvature of $M$ is at most $\kappa<0$. Then, for each $\epsilon>0$, there is a sweepout of the component of $M-S_{1}$ containing $S_{2}$, equivalent to the sweepout determined by $S_{2}$, so that each surface in this sweepout has area at most $\left(2 \pi\left|\chi\left(S_{2}\right)\right| /|\kappa|\right)+\epsilon$.

One has a good deal of geometric control over minimal surfaces when $M$ is negatively curved. As observed above, their area is bounded in terms of their Euler characteristic and the supremal sectional curvature of $M$. In fact, by ruling out the existence of long thin tubes in the surface, one has the following. Theorem 8. There is a function $f: \mathbb{R} \times \mathbb{R} \rightarrow \mathbb{R}$ with the following property. Let $M$ be a Riemannian 3-manifold, whose injectivity radius is at least $\epsilon / 2>0$, and whose sectional curvature is at most $\kappa<0$. Let $S$ be a closed minimal surface in $M$. Then there is a collection of at most $f(\kappa, \epsilon)|\chi(S)|$ points in $S$, such that the balls of radius $f(\kappa, \epsilon)$ about these points cover $S$. (Here, distance is measured using the path metric on $S$.)

This is proved in Proposition 6.1 of [3]. More precisely, formulas (1) and (2) there give the result.

\section{The Cheeger constant of manifolds and graphs}

The Cheeger constant of a compact Riemannian manifold $M$ is defined to be

$$
h(M)=\inf \left\{\frac{\operatorname{Area}(S)}{\min \left\{\operatorname{Volume}\left(M_{1}\right), \operatorname{Volume}\left(M_{2}\right)\right\}}\right\}
$$

where $S$ ranges over all embedded codimension one submanifolds that divide $M$ into $M_{1}$ and $M_{2}$.

A central theme of [3] is that the Cheeger constant of a 3-manifold and its Heegaard splittings are intimately related. One example of this phenomenon is the following result. 
Theorem 9. Let $M$ be a closed Riemannian 3-manifold. Let $\kappa<0$ be the supremum of its sectional curvatures. Then

$$
h(M) \leq \frac{4 \pi \chi_{-}^{h}(M)}{|\kappa| \text { Volume }(M)}
$$

This is essentially Theorem 4.1 of [3]. However, there, $\chi_{-}^{h}(M)$ is replaced by $c_{+}(M)$, which is an invariant defined in terms of the generalised Heegaard splittings of $M$. But the above inequality follows from an identical argument. We briefly summarise the proof.

From a minimal genus Heegaard splitting of $M$, construct a generalised Heegaard splitting $\left\{F_{1}, \ldots, F_{n}\right\}$ satisfying (1) to (5) of Theorem 3 . Let $\bar{F}$ be the surface obtained from $\bigcup_{j} F_{j}$ by discarding multiple copies of parallel components. Apply the isotopy of Theorem 5 to the incompressible components of $\bar{F}$. Each complementary region corresponds to a component of the complement of the even surfaces, and therefore contains a component of some odd surface $F_{j}$. Label this region with the integer $j$, and let $M_{j}$ be the union of the regions labelled $j$. There is some odd $j$ such that the volumes of $M_{1} \cup \cdots \cup M_{j-2}$ and $M_{j+2} \cup \cdots \cup M_{n}$ are each at most half the volume of $M$. Now, $F_{j} \cap M_{j}$ forms a strongly irreducible Heegaard surface for $M_{j}$. Applying Addendum 7 , we find for each $\epsilon>0$, a sweepout of $M_{j}$, equivalent to that determined by $F_{j}$, by surfaces with area at most $\left(2 \pi\left|\chi\left(F_{j}\right)\right| /|\kappa|\right)+\epsilon$. But, $\left|\chi\left(F_{j}\right)\right| \leq \chi_{-}^{h}(M)$, by (4) of Theorem 3. Some surface in this sweepout divides $M$ into two parts of equal volume. So, as $\epsilon$ was arbitrary,

$$
h(M) \leq \frac{4 \pi \chi_{-}^{h}(M)}{|\kappa| \text { Volume }(M)} .
$$

In this paper, we will consider the Cheeger constants of regular finite-sheeted covering spaces $M_{i}$ of $M$. Here, $M_{i}$ is given the Riemannian metric lifted from $M$. It is possible to estimate $h\left(M_{i}\right)$ in terms of graph-theoretic data, as follows.

By analogy with the Cheeger constant for a Riemannian manifold, one can define the Cheeger constant $h(X)$ of a finite graph $X$. If $A$ is a subset of the vertex set $V(X), \partial A$ denotes those edges with precisely one endpoint in $A$. Then $h(X)$ is defined to be

$$
\inf \left\{\frac{|\partial A|}{|A|}: A \subset V(X) \text { and } 0<|A| \leq|V(X)| / 2\right\}
$$

Proposition 10. Let $M$ be a compact Riemannian manifold. Let $\mathcal{X}$ be a finite set of generators for $\pi_{1} M$. Then there is a constant $k_{1} \geq 1$ with the following property. If $X_{i}$ is the Cayley graph of $\pi_{1} M / \pi_{1} M_{i}$ with respect to the generators $\mathcal{X}$, then

$$
k_{1}^{-1} h\left(X_{i}\right) \leq h\left(M_{i}\right) \leq k_{1} h\left(X_{i}\right) .
$$


This is essentially contained in [1], but we outline a proof. Lemma 2.3 of [3] states that, if $\mathcal{X}$ and $\mathcal{X}^{\prime}$ are two finite sets of generators for $\pi_{1} M$, then there is a constant $k \geq 1$ with the following property. If $X_{i}$ and $X_{i}^{\prime}$ are the Cayley graphs of $\pi_{1} M / \pi_{1} M_{i}$ with respect to $\mathcal{X}$ and $\mathcal{X}^{\prime}$, then

$$
k^{-1} h\left(X_{i}\right) \leq h\left(X_{i}^{\prime}\right) \leq k h\left(X_{i}\right) .
$$

Thus, for the purposes of proving Proposition 10, we are free to choose $\mathcal{X}$. We do this as follows. We pick a connected fundamental domain in the universal cover of $M$. The translates of this domain to which it is adjacent correspond to a finite set $\mathcal{X}$ of generators for $\pi_{1} M$. There is an induced fundamental domain in any finite regular cover $M_{i}$ of $M$. Its translates are in one-one correspondence with the group $\pi_{1} M / \pi_{1} M_{i}$. Two translates are adjacent if and only if one is obtained from the other by right-multiplication by an element in $\mathcal{X}$. Thus, the Cayley graph $X_{i}$ should be viewed as a coarse approximation to $M_{i}$. Any subset $A$ of $V\left(X_{i}\right)$, as in the definition of $h\left(X_{i}\right)$, therefore determines a decomposition of $M_{i}$. After a further modification, we may assume that this is along a codimension one submanifold. The existence of a constant $k_{1}$ such that $h\left(M_{i}\right) \leq k_{1} h\left(X_{i}\right)$ is then clear. The other inequality is more difficult to establish. One needs to control the geometry of a codimension one submanifold $S$ in $M_{i}$ that is arbitrarily close to realising the Cheeger constant of $M_{i}$. This is achieved in the proof of Lemma 2 of $[1]$.

\section{Constructing non-trivial cocycles}

Some new machinery has been developed in [3] that gives necessary and sufficient conditions on a finitely presented group to have finite index subgroups with infinite abelianisation. We describe some of the ideas behind this now.

Let $C$ be a finite cell complex with a single 0 -cell and in which every 2-cell is a triangle. Let $G$ be its fundamental group, and let $\mathcal{X}$ be the generators arising from the 1-cells. Associated with any finite index normal subgroup $H_{i}$ of $G$, there is a finite-sheeted covering space $C_{i}$ of $C$. Its 1-skeleton $X_{i}$ is the Cayley graph of $G / H_{i}$ with respect to $\mathcal{X}$. The following theorem is an expanded form of Lemma 2.4 of [3] and has exactly the same proof. It will play a key rôle in this paper.

Theorem 11. Suppose that $h\left(X_{i}\right)<\sqrt{2 /\left(3\left|V\left(X_{i}\right)\right|\right)}$. Let $A$ be any non-empty subset of $V\left(X_{i}\right)$ such that $|\partial A| /|A|=h\left(X_{i}\right)$ and $|A| \leq\left|V\left(X_{i}\right)\right| / 2$. Then there is a 1-cocycle $c$ on $C_{i}$ that is not a coboundary. Its support is a subset of the edges of $\partial A$, and it takes values in $\{-1,0,1\}$. As a consequence, $H_{i}$ has infinite abelianisation.

\section{The proof of the main theorem}

We start with a closed orientable 3 -manifold $M$ admitting a negatively curved Riemannian metric. After a small perturbation, we may assume that the metric is bumpy. Let $\kappa<0$ be the supremum of its sectional curvatures. Pick a 1-vertex 
triangulation $T$ of $M$. The edges of $T$, when oriented in some way, form a set $\mathcal{X}$ of generators for $\pi_{1}(M)$. Let $K$ be the 2-skeleton of the complex dual to $T$.

We will consider a collection $\left\{M_{i} \rightarrow M\right\}$ of finite regular covers of $M$, having the properties of Theorem 1. In particular, we will assume (at least) that $\chi_{-}^{h}\left(M_{i}\right) / \sqrt{d}_{i} \rightarrow 0$. (Note that this is justified when proving Theorem 1(2), by passing to a subsequence, and using the fact that $\chi_{-}^{h}\left(M_{i}\right) \leq \chi_{-}^{s h}\left(M_{i}\right)$.) The triangulation $T$ and 2-complex $K$ lift to $T_{i}$ and $K_{i}$, say, in $M_{i}$. The 1-skeleton of $T_{i}$ forms the Cayley graph $X_{i}$ of $G_{i}=\pi_{1} M / \pi_{1} M_{i}$ with respect to $\mathcal{X}$.

According to Theorem 9,

$$
h\left(M_{i}\right) \leq \frac{4 \pi}{|\kappa|} \frac{\chi_{-}^{h}\left(M_{i}\right)}{\operatorname{Volume}\left(M_{i}\right)}=\frac{4 \pi}{|\kappa| \operatorname{Volume}(M)} \frac{\chi_{-}^{h}\left(M_{i}\right)}{d_{i}},
$$

By Proposition 10, there is a constant $k_{1} \geq 1$ independent of $i$ such that $h\left(X_{i}\right) \leq$ $k_{1} h\left(M_{i}\right)$. Setting

$$
k_{2}=\frac{4 \pi k_{1}}{|\kappa| \operatorname{Volume}(M)},
$$

we deduce that

$$
h\left(X_{i}\right) \leq k_{2} \frac{\chi_{-}^{h}\left(M_{i}\right)}{d_{i}} .
$$

Let $V\left(X_{i}\right)$ be the vertex set of $X_{i}$. Let $A$ be a non-empty subset of $V\left(X_{i}\right)$ such that $|\partial A| /|A|=h\left(X_{i}\right)$ and $|A| \leq\left|V\left(X_{i}\right)\right| / 2=d_{i} / 2$. By Theorem 11, when $h\left(X_{i}\right)<\sqrt{2 /\left(3 d_{i}\right)}, T_{i}$ admits a 1-cocycle $c$ that is not a coboundary. Since $h\left(X_{i}\right) \leq k_{2} \chi_{-}^{h}\left(M_{i}\right) / d_{i}$, and we are assuming (at least) that $\chi_{-}^{h}\left(M_{i}\right) / \sqrt{d_{i}} \rightarrow 0$, then such a cocycle exists for all sufficiently large $i$. This establishes (1) of the Theorem 1.

Theorem 11 states that $c$ takes values in $\{-1,0,1\}$, and its support is a subset of the edges of $\partial A$. Dual to this cocycle is a transversely oriented normal surface $S$ in $T_{i}$ which is homologically non-trivial. Remove any 2 -sphere components from $S$. This is still homologically non-trivial, since all 2 -spheres in $M_{i}$ are inessential, as $M_{i}$ is negatively curved. The intersection of $S$ with the 2-skeleton of $T_{i}$ is a graph in $S$ whose complementary regions are triangles and squares. Let $V(S)$ and $E(S)$ be its vertices and edges. Its vertices are in one-one correspondence with the edges of $T_{i}$ in the support of $c$. So,

$$
|V(S)| \leq|\partial A|=|A| h\left(X_{i}\right) \leq d_{i} h\left(X_{i}\right) / 2 \leq k_{2} \chi_{-}^{h}\left(M_{i}\right) / 2 .
$$

The valence of each vertex is at most the maximal valence of an edge in $T, k_{3}$, say. So,

$$
|\chi(S)|<|E(S)| \leq|V(S)| k_{3} / 2 \leq k_{2} k_{3} \chi_{-}^{h}\left(M_{i}\right) / 4 .
$$

Setting $k_{4}=k_{2} k_{3} / 4$, we have deduced the existence of a homologically non-trivial, transversely oriented, properly embedded surface $S$ with $|\chi(S)| \leq k_{4} \chi_{-}^{h}\left(M_{i}\right)$ and with no 2-sphere components. By compressing $S$ and removing components if 
necessary, we may assume that $S$ is also incompressible and connected. Thus, we have proved the following result.

Theorem 12. Let $M$ be a closed orientable 3-manifold with a negatively curved Riemannian metric. Then there is a constant $k_{4}>0$ with the following property. Let $\left\{M_{i} \rightarrow M\right\}$ be a collection of finite regular covers, with degree $d_{i}$. If $\chi_{-}^{h}\left(M_{i}\right) / \sqrt{d_{i}} \rightarrow 0$, then, for all sufficiently large $i, M_{i}$ contains an embedded, connected, oriented, incompressible, homologically non-trivial surface $S$ such that $|\chi(S)| \leq k_{4} \chi_{-}^{h}\left(M_{i}\right)$.

By a theorem of Freedman, Hass and Scott [2] (Theorem 5 in this paper), there is an ambient isotopy taking $S$ to a minimal surface. We therefore investigate the coarse geometry of minimal surfaces in $M_{i}$.

Set $\epsilon / 2$ to be the injectivity radius of $M$. Let $f(\kappa, \epsilon)$ be the function from Theorem 8. Let $\tilde{K}$ be the lift of the 2-complex $K$ to the universal cover of $M$. Let $k_{5}$ be the maximum number of complementary regions of $\tilde{K}$ that lie within a distance $f(\kappa, \epsilon)$ of any point, and let $k_{6}=f(\kappa, \epsilon) k_{5}$.

Lemma 13. Let $S$ be a minimal surface in $M_{i}$. Then $S$ intersects at most $k_{6}|\chi(S)|$ complementary regions of $K_{i}$. Hence, running through any such region, there are at most $k_{6}|\chi(S)|$ translates of $S$ under the covering group action of $G_{i}$. Proof. By Theorem 8 , the number of balls of radius $f(\kappa, \epsilon)$ required to cover $S$ is at most $f(\kappa, \epsilon)|\chi(S)|$. The centre of each of these balls has at most $k_{5}$ complementary regions of $K_{i}$ within a distance $f(\kappa, \epsilon)$. So, $S$ intersects at most $k_{6}|\chi(S)|$ complementary regions of $K_{i}$. Each such region corresponds to an element of $G_{i}$. To prove the second half of the lemma, we may concentrate on the region corresponding to the identity. Then a translate $g S$ runs through here, for some $g$ in $G_{i}$, if and only if $S$ runs through the region corresponding to $g^{-1}$. Thus, there can be at most $k_{6}|\chi(S)|$ such $g$.

Proof of Theorem 1(2). Let $F$ be a strongly irreducible Heegaard surface in $M_{i}$ with $|\chi(F)|=\chi_{-}^{s h}\left(M_{i}\right)$. By Theorem 6 , there is an ambient isotopy taking it either to a minimal surface or to the double cover of a minimal non-orientable surface, with a small tube attached. So, by Lemma 13, $F$ intersects at most $k_{6} \chi_{-}^{s h}\left(M_{i}\right)$ complementary regions of $K_{i}$. Hence, by Lemma 13 , the number of copies of $S$ that $F$ intersects is at most $\left(k_{6} \chi_{-}^{s h}\left(M_{i}\right)\right)\left(k_{6} k_{4} \chi_{-}^{h}\left(M_{i}\right)\right)$. This is less than $d_{i}$ if $\chi_{-}^{s h}\left(M_{i}\right) / \sqrt{d_{i}}$ is sufficiently small. So there is a translate of $S$ which misses $F$. It then lies in a complementary handlebody of $F$. But this is impossible, since $S$ is incompressible. So, $\chi_{-}^{s h}\left(M_{i}\right) / \sqrt{d_{i}}$ is bounded away from zero.

Proof of Theorem 1(3). For ease of notation, let $x=\chi_{-}^{h}\left(M_{i}\right)$. Let $\left\{F_{1}, \ldots, F_{n}\right\}$ be a generalised Heegaard splitting for $M_{i}$, satisfying (1) - (5) of Theorem 3, obtained from a minimal genus Heegaard splitting. Replace any components of $F_{1} \cup \cdots \cup F_{n}$ that are parallel by a single component, and let $\bar{F}$ be the resulting surface. Isotope $\bar{F}$ so that each component is as in Theorems 5 or 6 . Corollary 4 states that $|\chi(\bar{F})|<x^{2}$. By Lemma 13, the number of complementary regions of $K_{i}$ that can intersect $\bar{F}$ is at most $k_{6} x^{2}$. Let $D$ be the corresponding subset 
of $G_{i}$.

Similarly, let $C$ be the subset of $G_{i}$ that corresponds to those complementary regions of $K_{i}$ which intersect $S$. By Lemma $13,|C| \leq k_{6}|\chi(S)| \leq k_{6} k_{4} x$.

We claim that, when $i$ is sufficiently large, there are at least $9 x / 2$ disjoint translates of $S$ under $G_{i}$ that are also disjoint from $\bar{F}$. Let $m=9 x / 2$. If the claim is not true, then for any $m$-tuple $\left(g_{1} S, \ldots, g_{m} S\right)$ of copies of $S$ (where $g_{j} \in G_{i}$ for each $j$ ), either at least two intersect or one copy intersects $\bar{F}$. In the former case, $g_{j} c_{1}=g_{k} c_{2}$, for some $c_{1}$ and $c_{2}$ in $C$, and for $1 \leq j<k \leq m$. Hence, $g_{k}^{-1} g_{j} \in C C^{-1}$. In the latter case, $g_{j} c_{1}=d$ for some $c_{1}$ in $C$ and $d$ in $D$, and so $g_{j} \in D C^{-1}$. Thus, the sets $q_{j k}^{-1}\left(C C^{-1}\right)$ and $p_{j}^{-1}\left(D C^{-1}\right)$ cover $\left(G_{i}\right)^{m}$, where $q_{j k}$ and $p_{j}$ are the maps

$$
\begin{aligned}
q_{j k}:\left(G_{i}\right)^{m} & \rightarrow G_{i} \\
\left(g_{1}, \ldots, g_{m}\right) & \mapsto g_{k}^{-1} g_{j} \\
p_{j}:\left(G_{i}\right)^{m} & \rightarrow G_{i} \\
\left(g_{1}, \ldots, g_{m}\right) & \mapsto g_{j},
\end{aligned}
$$

for $1 \leq j<k \leq m$. The former sets $q_{j k}^{-1}\left(C C^{-1}\right)$ each have size $\left|G_{i}\right|^{m-1}\left|C C^{-1}\right|$, and the latter sets $p_{j}^{-1}\left(D C^{-1}\right)$ have size $\left|G_{i}\right|^{m-1}\left|D C^{-1}\right|$. So,

$$
\left|G_{i}\right|^{m} \leq\left(\begin{array}{c}
m \\
2
\end{array}\right)\left|G_{i}\right|^{m-1}|C|^{2}+m\left|G_{i}\right|^{m-1}|C||D|
$$

This implies that

$$
d_{i}=\left|G_{i}\right| \leq\left(\begin{array}{c}
m \\
2
\end{array}\right)\left(k_{6} k_{4} x\right)^{2}+m\left(k_{6} k_{4} x\right)\left(k_{6} x^{2}\right)
$$

The right-hand side has order $x^{4}$ as $i \rightarrow \infty$. However, $x / \sqrt[4]{d_{i}} \rightarrow 0$, which is a contradiction, proving the claim.

Consider these $9 x / 2$ copies of $S$. Each lies in the complement of $\bar{F}$, which is a collection of compression bodies. Since $S$ is incompressible and connected, each copy of $S$ must be parallel to a component of $\bar{F}$. By Corollary $4(2), \bar{F}$ has at most $3 x / 2$ components. So, at least 3 copies of $S$ are parallel, and at least 2 of these are coherently oriented. The proof is now completed by the following lemma.

Lemma 14. Let $S$ be a connected, embedded, oriented, incompressible, nonseparating surface in a closed orientable 3-manifold $M_{i}$. Suppose that the image of $S$ under some finite order orientation-preserving homeomorphism $h$ of $M_{i}$ is disjoint from $S$, parallel to it and coherently oriented. Then $M_{i}$ fibres over the circle with fibre $S$.

Proof. Let $Y$ be the manifold lying between $S$ and $h(S)$. It is copy of $S \times I$, with $S$ and $h(S)$ corresponding to $S \times\{0\}$ and $S \times\{1\}$. Take a countable collection 
$\left\{Y_{n}: n \in \mathbb{Z}\right\}$ of copies of this manifold. Glue $S \times\{1\}$ in $Y_{n}$ to $S \times\{0\}$ in $Y_{n+1}$, via $h^{-1}$. The resulting space $Y_{\infty}$ is a copy of $S \times \mathbb{R}$. Let $H$ be the automorphism of this space taking $Y_{n}$ to $Y_{n+1}$ for each $n$, via the 'identity'. Let $p: Y_{0} \rightarrow Y$ be the identification homeomorphism. Extend this to a map $p: Y_{\infty} \rightarrow M_{i}$ by defining $p \mid Y_{n}$ to be $h^{n} p H^{-n}$.

We claim that this is a covering map. It may expressed as a composition $Y_{\infty} \rightarrow Y_{\infty} /\left\langle H^{N}\right\rangle \rightarrow M_{i}$, where $N$ is the order of $h$. The first of these maps is obviously a covering map. The second is also, since it is a local homeomorphism and $Y_{\infty} /\left\langle H^{N}\right\rangle$ is compact. Hence, $p$ is a covering map.

By construction, $h^{n}(S)$ lifts homeomorphically to $Y_{n-1} \cap Y_{n}$, for each $n$. Hence, the inverse image of $S$ in $Y_{\infty}$ includes all translates of $Y_{-1} \cap Y_{0}$ under $\left\langle H^{N}\right\rangle$. These translates divide $Y_{\infty}$ into copies of $S \times I$. Since $p^{-1}(S)$ is incompressible and any closed embedded incompressible surface in $S \times I$ is horizontal, we deduce that $p^{-1}(S)$ divides $Y_{\infty}$ into a collection of copies of $S \times I$. The restriction of $p$ to one of these components $Z$ is a covering map to a component of $M_{i}-S$. But $M_{i}-S$ is connected, as $S$ is connected and non-separating. So, $p$ maps $Z$ surjectively onto $M_{i}-S$. By examining this map near $S$, we see that it is degree one and hence a homeomorphism. Therefore, $M_{i}$ is obtained from a copy of $S \times I$ by gluing its boundary components homeomorphically. So, $M_{i}$ fibres over the circle with fibre $S$.

\section{References}

[1] R. Brooks, The spectral geometry of a tower of coverings, J. Differential Geom. 23 (1986) 97-107.

[2] M. Freedman, J. Hass and P. Scott, Least area incompressible surfaces in 3-manifolds, Invent. Math. 71 (1983) 609-642.

[3] M. Lackenby, Heegaard splittings, the virtually Haken conjecture and Property $(\tau)$, Preprint.

[4] A. Lubotzky, Eigenvalues of the Laplacian, the first Betti number and the congruence subgroup problem, Ann. Math. (2) 144 (1996) 441-452.

[5] J. Pitts and J. H. Rubinstein, Existence of minimal surfaces of bounded topological type in three-manifolds, Miniconference on geometry and partial differential equations (Canberra, 1985), 163-176.

[6] M. Scharlemann, Heegaard splittings, Handbook of Geometric Topology (Elsevier, 2002), 921-953.

[7] M. Scharlemann and A. Thompson, Thin position for 3-manifolds, Geometric topology (Haifa, 1992), 231-238, Contemp. Math., 164.

[8] B. White, The space of minimal submanifolds for varying Riemannian metrics, Indiana Math. Journal 40 (1991) 161-200.

Mathematical Institute, Oxford University, 24-29 St Giles', Oxford OX1 3LB, United KINGDOM

E-mail address: lackenby@maths.ox.ac.uk 